OPEN ACCESS

Edited by:

Yurong Lai,

Gilead, United States

Reviewed by:

Constantin Mircioiu,

Carol Davila University of Medicine and Pharmacy, Romania

Stanislav Yanev,

Institute of Neurobiology (BAS),

Bulgaria

*Correspondence:

Jiyue Cao

caojiyue2@163.com

Qigai He

he628@mail.hzau.edu.cn

Specialty section:

This article was submitted to Drug Metabolism and Transport,

a section of the journal

Frontiers in Pharmacology

Received: 18 May 2018

Accepted: 22 June 2018

Published: 26 July 2018

Citation

Lei Z, Liu Q, Qi Y, Yang B, Khaliq H, Xiong J, Moku GK, Ahmed S, Li K, Zhang $\mathrm{H}$, Zhang $W$, Cao $J$ and $H e Q$ (2018) Optimal Regimens and Cutoff Evaluation of Tildipirosin Against Pasteurella multocida.

Front. Pharmacol. 9:765. doi: 10.3389/fphar.2018.00765

\section{Optimal Regimens and Cutoff Evaluation of Tildipirosin Against Pasteurella multocida}

\author{
Zhixin Lei ${ }^{1,2,3,4}$, Qianying Liu' ${ }^{1,2,3}$, Yi Qi ${ }^{1}$, Bing Yang ${ }^{1,2}$, Haseeb Khaliq ${ }^{1}$, Jincheng Xiong ${ }^{1,2}$,

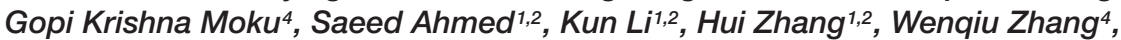 \\ Jiyue $\mathrm{CaO}^{2,3 *}$ and Qigai $\mathrm{He}^{1 *}$
}

${ }^{1}$ State Key Laboratory of Agricultural Microbiology, College of Veterinary Medicine, Huazhong Agricultural University, Wuhan, China, ${ }^{2}$ Department of Veterinary Pharmacology, College of Veterinary Medicine, Huazhong Agricultural University, Wuhan, China, ${ }^{3}$ National Reference Laboratory of Veterinary Drug Residues and MAO Key Laboratory for Detection of Veterinary Drug Residues, Huazhong Agricultural University, Wuhan, China, ${ }^{4}$ Department of Pharmaceutics, College of Pharmacy, University of Minnesota, Minneapolis, MN, United States

Pasteurella multocida (PM) can invade the upper respiratory tract of the body and cause death and high morbidity. Tildipirosin, a new 16-membered-ring macrolide antimicrobial, has been recommended for the treatment of respiratory diseases. The objective of this research was to improve the dose regimes of tildipirosin to PM for reducing the macrolides resistance development with the pharmacokinetic/pharmacodynamic (PK/PD) modeling approach and to establish an alternate cutoff for tildipirosin against PM. A single dose (4 mg/kg body weight) of tildipirosin was administered via intramuscular (i.m.) and intravenous (i.v.) injection to the pigs. The minimum inhibitory concentration (MIC) values of clinical isolates (112) were measured in the range of $0.0625-32 \mu \mathrm{g} / \mathrm{ml}$, and the $\mathrm{MIC}_{50}$ and $\mathrm{MIC}_{90}$ values were 0.5 and $2 \mu \mathrm{g} / \mathrm{ml}$, respectively. The MIC of the selected PM04 was 2 and $0.5 \mu \mathrm{g} / \mathrm{ml}$ in the tryptic soy broth (TSB) and serum, respectively. The main pharmacokinetic (PK) parameters including the area under the curve at $24 \mathrm{~h}\left(\mathrm{AUC}_{24} \mathrm{~h}\right), \mathrm{AUC}$, terminal half-life $\left(T_{1 / 2}\right)$, the time to peak concentration $\left(T_{\max }\right)$, peak concentration $\left(C_{\max }\right)$, relative total systemic clearance $\left(\mathrm{CL}_{\mathrm{b}}\right)$, and the last mean residence time (MRT last) were calculated to be 7.10, $7.94 \mu g^{*} \mathrm{~h} / \mathrm{ml}, 24.02$, NA h, NA $\mu \mathrm{g} / \mathrm{ml}, 0.46 \mathrm{~L} / \mathrm{h}^{*} \mathrm{~kg}, 8.06 \mathrm{~h}$ and 3.94, $6.79 \mu \mathrm{g}^{*} \mathrm{~h} / \mathrm{ml}, 44.04,0.25 \mathrm{~h}, 0.98 \mu \mathrm{g} / \mathrm{ml}, 0.43 \mathrm{~L} / \mathrm{h} * \mathrm{~kg}, 22.85 \mathrm{~h}$ after i.v. and i.m. induction, respectively. Moreover, the bioavailability of i.m. route was $85.5 \%$, and the unbinding of tildipirosin to serum protein was $78 \%$. The parameters $A C_{24} h / M I C$ in serum for bacteriostatic, bactericidal, and elimination activities were calculated as 18.91, 29.13, and $34.03 \mathrm{~h}$ based on the inhibitory sigmoid $E_{\max }$ modeling. According to the Monte Carlo simulation, the optimum doses for bacteriostatic, bactericidal, and elimination activities were $6.10,9.41$, and $10.96 \mathrm{mg} / \mathrm{kg}$ for $50 \%$ target and 7.86 , 12.17 , and $14.57 \mathrm{mg} / \mathrm{kg}$ for $90 \%$ target, respectively. The epidemiological cutoff value (ECV) was calculated to be $4 \mu \mathrm{g} / \mathrm{ml}$ which could cover $95 \%$ wild-type clinical isolates distribution. The PK-PD cutoff (COPD) was analyzed to be $0.25 \mu \mathrm{g} / \mathrm{ml}$ in vitro for tildipirosin against PM based on the Monte Carlo simulation. Compared with these 
two cutoff values, the finial susceptible breakpoint was defined as $4 \mu \mathrm{g} / \mathrm{ml}$. The data presented now provides the optimal regimens $(12.17 \mathrm{mg} / \mathrm{kg})$ and susceptible breakpoint ( $4 \mu \mathrm{g} / \mathrm{ml}$ ) for clinical use, but these predicted data should be validated in the clinical practice.

Keywords: Pasteurella multocida, tildipirosin, pharmacokinetic/pharmacodynamic, epidemiological cutoff, PKPD cutoff, optimal regimens

\section{INTRODUCTION}

Pasteurella multocida (PM) is a widespread pathogenic bacterium that can cause mucosal surfaces and respiratory tract infection in animals, which results in large economic losses in the livestock and poultry industry (Holst et al., 1992; Elazab et al., 2018). Furthermore, there are a few reports on the resistance of PM response to macrolide including tildipirosin, tilmicosin, tylosin, etc. (Andersen et al., 2012; Michael et al., 2012; Poehlsgaard et al., 2012). Therefore, it is essential for veterinarians to use antibiotics wisely in veterinary clinics.

Tildipirosin, a new 16-membered-ring macrolide, is a semisynthetic tylosin developed to treat respiratory pathogens, such as HPS, P. multocida (PM), APP, MH (Rose et al., 2013; Torres et al., 2016). With widespread use of macrolides, such as tylosin, tilmicosin, and tildipirosin in Europe, a new macrolide tildipirosin-resistant PM has emerged (Andersen et al., 2012; Olsen et al., 2015). Furthermore, tildipirosin was imported into China, and resistant strains were found in the clinical setting, breeding, and animal husbandry.

It is better to set the susceptibility of target bacteria to the antibiotics to reduce the development of bacterial resistance and strengthen the management of antibiotic use. According to the guidance of the VetCAST (an EUCAST sub-committee for veterinary antimicrobial susceptibility testing), the susceptibility cutoff values consisted of an ECV - the highest MIC (>95\%) in the wild-type distribution; a $\mathrm{PK} / \mathrm{PD}$ cutoff value $\left(\mathrm{CO}_{\mathrm{PD}}\right)-$ the most probable critical value $(>90 \%)$ in the target population from the calculated PK/PD index such as AUC/MIC or terminal life $(\mathrm{T})>$ MIC; and a clinical cutoff - the curving ratios for the antibiotic against the target bacterium if it is possible to implement and obtain the statistical data (Toutain et al., 2017). Based on the guidance of CLSI and EUCAST, there has not been a recommended susceptibility breakpoint for tildipirosin against PM. Therefore, it would be of great significance to establish such

\footnotetext{
Abbreviations: APP, Actinobacillus pleuropneumoniae; AUC, the area under the curve; $\mathrm{AUC}_{24} \mathrm{~h}$, area under the curve at $24 \mathrm{~h}$; $\mathrm{CFU}$, colony forming unit; $\mathrm{CL}_{\mathrm{b}}$, relative total systemic clearance; CLSI, Clinical and Laboratory Standard Institute; $C_{\max }$, peak concentration; $\mathrm{CO}_{\mathrm{PD}}, \mathrm{PK} / \mathrm{PD}$ cutoff; ECVs, epidemiological cutoff values; EMA, European medicines agency; EUCAST, European Committee on Antimicrobial Susceptibility Testing; HPLC, high-performance liquid chromatography; HPS, Haemophilus parasuis; i.m., intramuscular; i.v., intravenous injection; $\mathrm{MBC}$, minimal bactericidal concentration; MH, Mannheimia haemolytica; MIC, minimum inhibitory concentration; MRT, the mean residence time; NCS, newborn calf-serum; PCR, polymerase chain reaction; $\mathrm{PD}$, pharmacodynamic; PK, pharmacokinetic; PK/PD, pharmacokinetics/pharmacodynamics; PM, Pasteurella multocida; PTA, the probability of target attainment; $T_{1 / 2}$, terminal half-life; TAR, target achievement ratio; $T_{\max }$, the time to peak concentration; TSB, tryptic soy broth; VetCAST, Veterinary Committee on Antimicrobial Susceptibility Testing.
}

a criterion for both susceptibility testing and monitoring the development of resistance (Zhixin et al., 2017; Lei et al., 2018a).

Misuse and unreasonable dosage of antimicrobial agents were the main factors for the development of resistance (Burgess, 1999; Burgess et al., 1999; Wang et al., 2016). However, PK/PD integration modeling data can provide an optimal drug dosage strategy, reducing resistance development, which is a key method to evaluate the clinically relevant relationship between time, drug concentration, and effect. In the present study, tildipirosin recommended dosage ( $4 \mathrm{mg} / \mathrm{kg}$ body weight) has been anticipated by the European medicines agency (EMA, $2010)$ and has also been verified in different doses (2, 4, and $6 \mathrm{mg} / \mathrm{kg}$ body weight) according to the PK data (area under concentration-time curve from time, $\left.\mathrm{AUC}_{\text {last }}\right)$ in the previous report (Menge et al., 2012; Rose et al., 2013). However, there was no data that supported the recommended dosage $(4 \mathrm{mg} / \mathrm{kg}$ body weight) under PD of tildipirosin against PM in China. This study investigated the $\mathrm{PK}$ and in vitro $\mathrm{PD}$ activity of tildipirosin in plasma obtained from healthy pigs, reviewed the recommended dosage, and formulated an optimum dose.

The objectives of the current study were (i) to assay the PD parameters and actions of tildipirosin against PM, (ii) to evaluate the PK properties of tildipirosin in plasma after i.m. administration, (iii) to formulate a rational dosage strategy and review the preceding recommended dosage based on PK/PD modeling for tildipirosin against PM, providing peak efficacy and marginal opportunity for the resistant development of PM (Jian et al., 2015; Xiao et al., 2015; Wang et al., 2016), and (iv) to establish the ECV and $\mathrm{CO}_{\mathrm{PD}}$ of tildipirosin against PM based on the wild-type MIC distributions and PK/PD profiles data in vitro and in vivo.

\section{MATERIALS AND METHODS}

\section{Bacterial Strain Isolation}

112 PM isolates were collected from pigs across China (mainly, Anhui, Hubei, Henan, Guangdong, and Jiangxi provinces) from 2013 to 2016. According to the MIC $_{90}$ values of strains, a PM named PM04 strain whose minimal inhibitory concentration (MIC) was similar to $\mathrm{MIC}_{90}$, was considered to find the antimicrobial activity of tildipirosin in vitro. Escherichia coli ATCC 25922 isolate was selected as the reference strain for determining antibiotic susceptibility. The isolate species were recognized by the method of PCR. Before testing MIC values, subculturing was performed for each sample in TSB and tryptic soy agar (TSA; Qingdao Hai Bo Biological Technology Co., Ltd., Shandong, China) containing 5\% NCS (Zhejiang Tianhang Biotechnology Co., Ltd., Zhejiang, China) at least 
thrice. Tildipirosin was provided by Hubei Huisheng Biological Technology Company (Hubei, China).

\section{Determination of MIC and MBC}

The MICs of 112 PM were measured with the process of agar dilution technique in line with the CLSI guidelines (CLSI, 2012; Zhang et al., 2016). Strains of PM $\left(2-4 \mu \mathrm{l}\right.$, about $\left.10^{8} \mathrm{CFU} / \mathrm{ml}\right)$ were administrated onto TSA agar plates containing NCS, with twofold serial-dilutions of tildipirosin $(0.0625-32 \mu \mathrm{g} / \mathrm{ml})$. The plates with strains were nurtured in the atmosphere containing $5 \% \mathrm{CO}_{2}$ for $48 \mathrm{~h}$ at $37^{\circ} \mathrm{C}$. The MIC contained a minimum amount of tildipirosin where the visible growth of bacteria was inhibited.

The $100 \mu$ l suspension from the 96 well plates of PM04, of which MIC amount was estimated in broth dilution technique according to the CLSI guidelines, was subjected to 10 -fold or more dilution with TSB, and then $10 \mu \mathrm{l}$ of each diluted suspension was spread on the TSA plates and the colony counts were calculated after containing $5 \% \mathrm{CO}_{2}$ for $48 \mathrm{~h}$ at $37^{\circ} \mathrm{C}$. $\mathrm{MBC}$ was the minimum concentration of tildipirosin inhibiting $99.9 \%$ bacterial density of PM.

\section{ECV Determination for Tildipirosin Against PM}

The resistance bacteria were excluded, and the wild-type PM should be defined for microorganisms which did not have any acquired resistance mechanisms to the tildipirosin. Additionally, the ECV calculated as the peak MIC for wild-type PM comprised over $95 \%$ in the MIC distributions according to the guidelines of CLSI and the previously described reports (Turnidge and Paterson, 2007; Pfaller et al., 2010, 2011; Lei et al., 2018a). The wild-type MIC distributions were checked and amended on the basis of standard distribution at the lower end of the MIC range for attaining the suitable distribution of MICs, which was executed with Sigma-Stat software (version 3.5, Systat Software Inc., United States). The mean and standard deviation of the normal distribution for the optimum non-linear least squares regression fitting MICs were assessed based on the software of GraphPad Prism (Version 7, GraphPad Software Inc., United States). The final ECV was calculated as the MIC value that captured over $95 \%$ of the optimum MIC distributions using the NORMINV and NORDIST functions in Microsoft Excel software was based on the preceding mean and standard deviation values. In other words, the ECV could be obtained using a summarized Excel form based on the above method.

\section{Time-Killing Curves in Vitro and ex Vivo}

According to the MIC of tildipirosin against PM04 in MIC value, TSA plates were made with various concentrations of tildipirosin from the range of $1 / 4$ to $32 \mathrm{MIC}$ detailed in the previous study by Zhang and Lei and their companions (Zhang et al., 2016; Lei et al., 2018a). From the bacterial fluid, $100 \mu \mathrm{l}$ was diluted with normal sterile saline $\left(10^{-1}\right.$ to $10^{-5}$ dilution ratio), then aliquots of the last four diluted samples were plunged onto the TSA plates at $0,2,4,6,8,10,12$, and $24 \mathrm{~h}$ of culture, which were incubated in the atmosphere having $\mathrm{CO}_{2}$ for $48 \mathrm{~h}$ at $37^{\circ} \mathrm{C}$.
Serum obtained from the pigs was considered as a culturemedium for the ex vivo growth of isolates, MIC, and time-killing curve. The determination methods were similar to in vitro protocol mentioned above utilizing the serum as a substitute for the TSB method. The bacteria $\left(10^{6} \mathrm{CFU} / \mathrm{ml}\right)$ were co-incubated with the content of ileum samples collected from the pigs at various time intervals $(0,0.25,1,2,4,12$, and $24 \mathrm{~h})$ after supplementation with $4 \mathrm{mg} / \mathrm{kg}$ tildipirosin by i.m. route of administration. The ex vivo time-killing curve was fitted to a PD model with the theory of a decrease in tildipirosin concentration based on incubation time with inhibitory sigmoid $E_{\max }$ model.

\section{PK Study}

\section{Animals}

Eight healthy pigs (of both sexes), weighing $15-20 \mathrm{~kg}$ and $4-$ 5 weeks, were selected for this research work. These healthy animals were kept in a separate pen with free and adequate water availability, and no antibiotic feed was permitted. Moreover, animals were offered feed and water for 7 days to acclimate prior to the testing. After 7 days, pigs were intravenously (i.v.) injected with a single dose of $4 \mathrm{mg} / \mathrm{kg}$ tildipirosin. After a period of radical washing for approximately 2 weeks, the pigs were injected i.m. with the same dose of tildipirosin.

All animal experiments were permitted by Laboratory Animal Use and Care Committee in Hubei Science and Technology Agency (permit number SYXK 2013-0044) and executed according to the guidelines of committee. The anesthetics were given to reduce the pain and adverse effects in animals.

\section{Collection of Plasma Samples and HPLC Analysis}

Three $\mathrm{ml}$ of blood samples were collected at 15 and $30 \mathrm{~min}$, and then at various time points $-1,2,4,6,8,10,12,24,48$, and $72 \mathrm{~h}$, after i.v. and i.m. administrations. The obtained plasma samples were immediately freezed and then centrifuged at about $3000 \mathrm{~g}$ for $10 \mathrm{~min}$ before preserving them at $-80^{\circ} \mathrm{C}$ until further investigation.

A C18 reverse-phase column $(250 \mathrm{~mm} \times 4.6 \mathrm{~mm}$, i.d., $5 \mu \mathrm{m}$, Agilent, United States) was used for HPLC, which was performed with a $289 \mathrm{~nm}$ detection wavelength at $30^{\circ} \mathrm{C}$. Plasma $(0.5 \mathrm{ml})$ samples were mixed with $200 \mu \mathrm{l}$ dipotassium hydrogen phosphate solution $(0.1 \mathrm{~mol} / \mathrm{L})$, and then were extracted twice with $5 \mathrm{ml}$ diethyl ether. The supernatants were attained by the centrifugation method, and then vaporized to dryness under the nitrogen chamber at the water bath kettle with $45^{\circ} \mathrm{C}$. The final samples were re-suspended with the mobile phase by the initial volume $(0.5 \mathrm{ml})$. The samples for tildipirosin concentration determination were analyzed using HPLC method, which had been optimized by our laboratory in the previously described report (Lei et al., 2018a) within a month. The PK data were analyzed with Phoenix WinNonlin 6.1 software (Pharsight Co., Ltd.).

\section{Binding of Tildipirosin to Serum Protein}

The serum protein binding of tildipirosin was determined on the collected samples at different points from the eight pigs used in 
this study. The total concentration of tildipirosin was detected in every sample. The samples were redetermined after filtration through dialysis tubing (Thermo Fisher Scientific Co., LTD., United States).

\section{PK/PD Integration Analysis}

Although most macrolides were classified as time-dependent killing drugs, tildipirosin was concentration-dependent, and the PK/PD index were the $\mathrm{AUC}_{24} \mathrm{~h} / \mathrm{MIC}$ and the $C_{\max } / \mathrm{MIC}$ (Mouton et al., 2002; Chigutsa et al., 2012; Rose et al., 2013; Xiao et al., 2015). The $\mathrm{AUC}_{24} \mathrm{~h} / \mathrm{MIC}$ and $C_{\max } / \mathrm{MIC}$ were considered as paired $\mathrm{PK} / \mathrm{PD}$ parameters which were calculated in each dose of the time-killing curve. The inhibitory sigmoid $E_{\max }$ model was applied to evaluate the assimilation of correlation of $\mathrm{AUC}_{24} \mathrm{~h} / \mathrm{MIC}$ ratio in vitro and bacteria count change $(\mathrm{CFU} / \mathrm{ml})$ in serum during $24 \mathrm{~h}$ incubation with WinNonlin software (Aliabadi and Lees, 2000, 2001, 2002; Sidhu et al., 2011). The model equation was described as follows in equation 1.

$$
E=E_{\max } \cdot\left(1-\frac{\mathrm{C}^{\mathrm{N}}}{\mathrm{C}^{\mathrm{N}}+\mathrm{EC}_{50}^{\mathrm{N}}}\right)
$$

$E$, presented the effect of antimicrobial agent counted as $\log _{10}$ difference of bacterial number before and after the $24 \mathrm{~h}$ incubation in vitro; $E_{\max }$, measured the deviations in $\log _{10}$ difference between 0 and $24 \mathrm{~h}$ in the control and tildipirosin samples; $\mathrm{EC}_{50}$, the $\mathrm{AUC}_{24} / \mathrm{MIC}$ value reached $50 \%$ of the $E_{\max } ; \mathrm{C}$, presented the $\mathrm{AUC}_{24} / \mathrm{MIC}$ ratio; $N$, presented the Hill coefficient.

\section{Dose Estimations}

The given formula was performed to calculate the doses in different magnitudes of efficiency containing ( $E=0$, no change in bacterial count, $E=-1,99.9 \%$ reduction in the count, $E=-3$, 99.99\% reduction) for estimating an optimum regimen.

$$
\text { Dose }=\frac{(A U C / M I C) \cdot M I C_{90} \cdot C L}{f u \cdot F}
$$

AUC/MIC, meant the targeted endpoint for optimal efficacy; $M I C$, meant minimum inhibitory concentration; $C L$, meant clearance per day; $f u$, meant the free fraction of drug in plasma, ignoring if there was minimal binding; $F$, meant the bioavailability.

The distribution probabilities for predicted daily dosage were performed to achieve simulated 50 and 90\% TAR under 10,000 trails with Crystal Ball software (version 7.2.2, Oracle, United States) for bacteriostatic, bactericidal, and elimination activities.

\section{Monte Carlo Analysis and PK/PD Cutoff Calculation}

The Monte Carlo simulation (including 10,000 iterations) was performed using the Crystal Ball software (version 7.2.2, Oracle, United States) based on the selected $\mathrm{PK} / \mathrm{PD}$ target index (AUC ${ }_{24} / \mathrm{MIC}, E=-3$, bactericidal activity) (Lei et al., 2017a,b,c, 2018b). The COPD was calculated as the MIC when PTA reached up to $90 \%$ based on the CLSI guidelines and other previously described studies (Turnidge and Paterson, 2007; Lei et al., 2018a).

\section{Statistical Analysis}

$\mathrm{MIC}_{90}$ was measured by using statistical package of SPSS software, and statistical analysis was executed with Student's $t$-test. Significant differences were checked using $p<0.05$.

\section{RESULTS}

\section{MIC and MBC Determination Both in Vitro and ex Vivo}

The MIC distributions of tildipirosin against 112 clinical isolates of PM were displayed in Figure 1, of which MIC values ranged from 0.0625 to $32 \mu \mathrm{g} / \mathrm{ml} \mathrm{MIC}_{50}$ and $\mathrm{MIC}_{90}$ of the distribution were 0.5 and $2 \mu \mathrm{g} / \mathrm{ml}$, respectively, calculated with SPSS Statistics version 17.0, evincing that tildipirosin had a potent antibacterial efect on PM (Table 1).

According to the $\mathrm{MIC}_{90}$ value, a clinical isolate and PM04 whose MIC was similar to $\mathrm{MIC}_{90}$ was selected to explore the antimicrobial activity of tildipirosin in vitro. The MIC of tildipirosin against PM04 was $0.5 \mu \mathrm{g} / \mathrm{ml}$ which was 0.25 times lower than that $(2 \mu \mathrm{g} / \mathrm{ml})$ in TSB. The MBC of tildipirosin against PM04 in TSB and serum were 8 and $1 \mu \mathrm{g} / \mathrm{ml}$, respectively (Table 1 ). The ratios of $\mathrm{MBC} / \mathrm{MIC}$ in vitro (TSB) and ex vivo (serum) were 2 and 4 , respectively. Which indicated that tildipirosin might have a strong bacteriostatic activity both

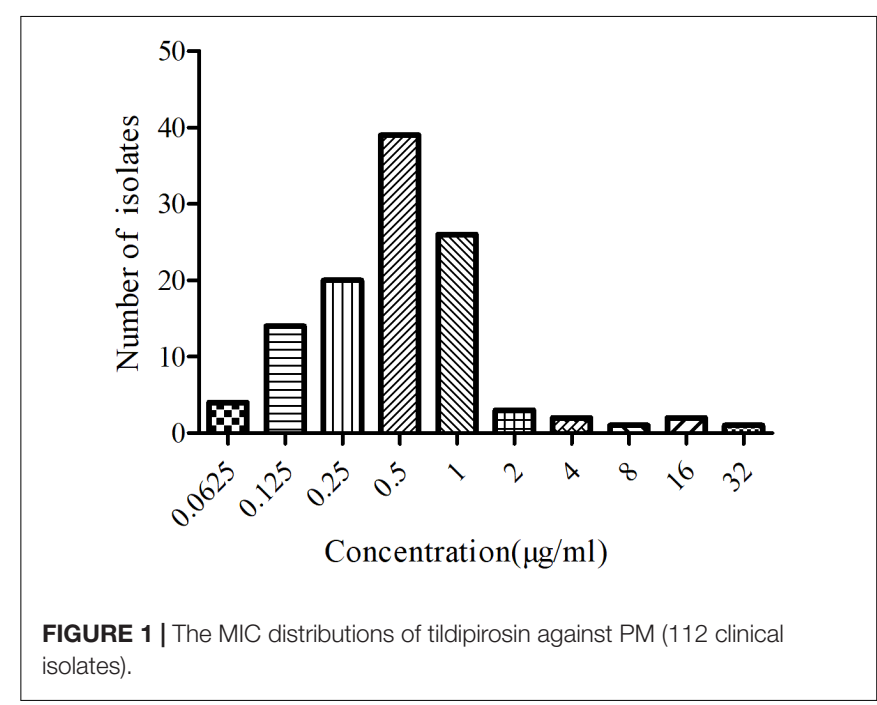

TABLE 1 | Minimum inhibitory concentration (MIC) and MBC $(\mu \mathrm{g} / \mathrm{ml})$ of tildipirosin against PMO4 in vitro and ex vivo.

\begin{tabular}{lcccc}
\hline Target strain & MIC & MBC & MIC $_{\mathbf{5 0}}$ & MIC $_{\mathbf{9 0}}$ \\
\hline TSB & 2 & 8 & 0.5 & 2 \\
Serum & 0.5 & 1 & - & -
\end{tabular}



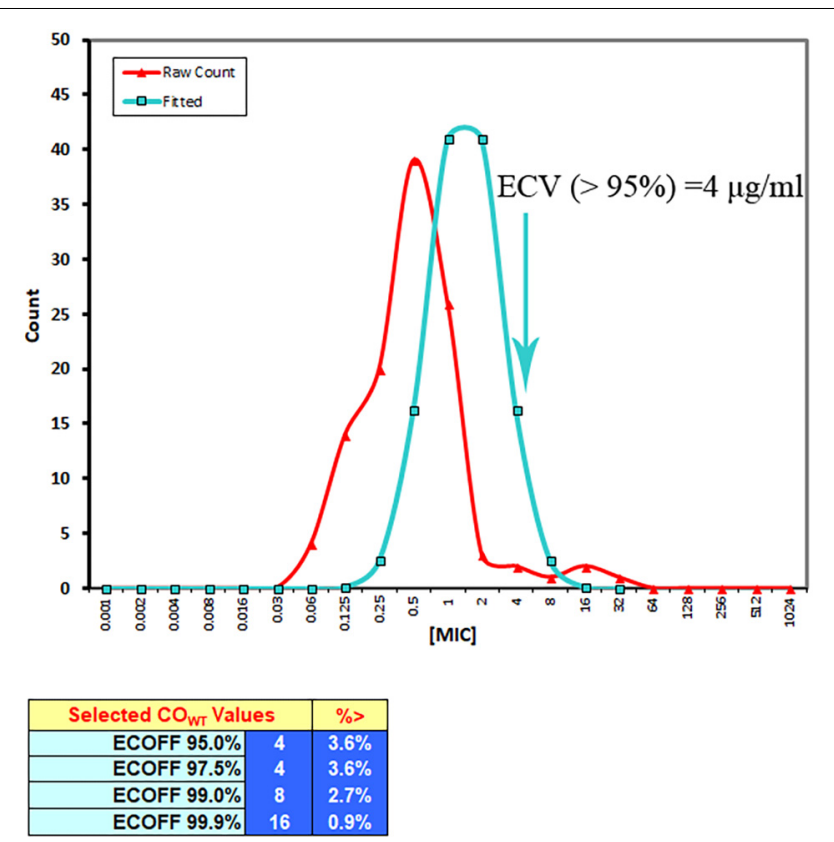

FIGURE 2 | The ECV evaluation using the statistical program "ECOFFinder."

in vitro and ex vivo, and concentration-dependent profile against PM.

\section{ECV Calculation}

The MIC distributions of tildipirosin against clinical PM were presented in Figure 1. According to the guidance of CLSI and the previously described reports (Turnidge and Paterson, 2007; Pfaller et al., 2011; Lei et al., 2018a), the resistant bacteria among the wild-type clinical isolates should be removed, therefore the three isolates whose MICs were over $16 \mu \mathrm{g} / \mathrm{ml}$ and detected to be the resistant bacteria should be discarded as reported by Michael et al. (2012). The wild-type MIC distributions were statistically detected by the calculation of over 95\% ECV plotted in Figure 2 using the freeware statistical program "ECOFFinder" which could estimate the wild-type population and derive the ECV value ${ }^{1}$. This software could simplify the operations and calculation procedures easily. The evaluated MICs whose values were 4,8 , and $16 \mu \mathrm{g} / \mathrm{ml}$ could encompass 97.5, 99, and 99.9\% of the wild-type isolates. Finally, the probability of the MIC at $4 \mu \mathrm{g} / \mathrm{ml}$ encompassed over $95 \%$ of the wild-type isolates and was defined as the ECV (Figure 2).

\section{Antimicrobial Activity in Vitro and ex Vivo}

Time-killing curves of tildipirosin against PM04 in vitro were illustrated in Figure 3. According to the curves profiles, tildipirosin displayed a concentration-dependent characteristic, a more rapid bactericidal activity with an increase of drug concentration. It acted intensively with bacteriostatic effect when the tildipirosin concentrations were higher than 2 MIC in vitro (TSB), while the concentration at $0.25 \mathrm{~h}(1 \mu \mathrm{g} / \mathrm{ml}, 2 \mathrm{MIC})$ could

${ }^{1}$ http://www.uphs.upenn.edu/bugdrug/antibiotic_manual/ECV.htm
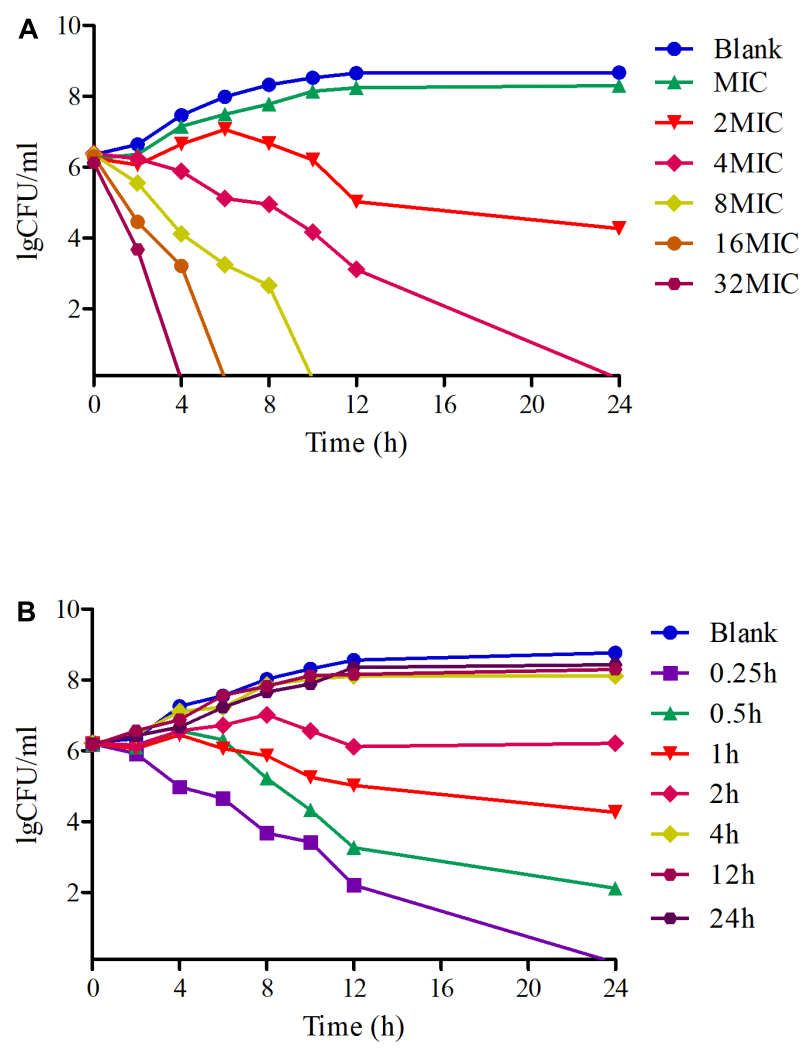

FIGURE 3 | Time-killing curves of tildipirosin against PMO4 in the TSB and serum. (A) Means the curve in the TSB (vitro), (B) means the curve in the serum (ex vivo).

eradicate PM04 completely in vivo (serum) (Figure 3). These results were similar to the $\mathrm{MBC}$ determination, but the difference of antimicrobial activity in TSB and serum might attribute to the effect of serum, which could have a positive antibacterial effect.

\section{PK of Tildipirosin}

The proposed HPLC method for tildipirosin was according to the previously described research in this lab and the detailed introduction is referred to in the report by Lei et al. (2018a). The PK parameters of tildipirosin in the serum after i.v. and i.m. administrations at a dose of $4 \mathrm{mg} / \mathrm{kg}$ were presented in Table 2. The PK parameters were derived by non-compartmental analysis. The PK parameters of tildipirosin in the serum were measured using WinNonlin software. The results for the $\mathrm{AUC}_{24} \mathrm{~h}, \mathrm{AUC}, T_{\max }, T_{1 / 2}$ of tildipirosin, $C_{\max }, \mathrm{CL}_{\mathrm{b}}$, and the mean resistance time (MRT) in the serum after i.v. and i.m. administrations were shown in Table 2. The bioavailability for tildipirosin after i.m. administration was determined as $85.5 \%$ (Table 2). The mean \pm SD of tildipirosin concentrationtime profiles were shown in Figure $\mathbf{4}$ after i.v. and i.m. administrations, respectively. Additionally, the percentage of free tildipirosin concentration with unbinding protein in the plasma was $78.00 \pm 5.23 \%$ from the eight pigs used in this study. 
TABLE 2 | Pharmacokinetic parameters in plasma after $4 \mathrm{mg} / \mathrm{kg}$ i.v. and i.m. administration, respectively.

\begin{tabular}{lccc}
\hline Parameters & Units & i.v & i.m \\
\hline $\mathrm{AUC}_{24} \mathrm{~h}$ & $\mu \mathrm{g}^{*} \mathrm{~h} / \mathrm{ml}$ & $7.10 \pm 0.91$ & $3.94 \pm 0.62$ \\
$\mathrm{AUC}$ & $\mu \mathrm{g}^{*} \mathrm{~h} / \mathrm{ml}$ & $7.94 \pm 1.11$ & $6.79 \pm 0.45$ \\
$T_{\max }$ & $\mathrm{h}$ & - & $0.25 \pm 0.06$ \\
$T_{1 / 2}$ & $\mathrm{~h}$ & $24.02 \pm 3.12$ & $44.04 \pm 4.56$ \\
$C_{\max }$ & $\mu \mathrm{g} / \mathrm{ml}$ & - & $0.98 \pm 0.11$ \\
$\mathrm{CL}_{\mathrm{b}}$ & $\mathrm{h}$ & $0.46 \pm 0.08$ & $0.43 \pm 0.07$ \\
$\mathrm{MRT}_{\text {last }}$ & $\mathrm{h}$ & $8.06 \pm 1.6$ & $22.85 \pm 2.96$ \\
$F$ & $\%$ & & $85.5 \pm 7.11$
\end{tabular}

$A \cup C_{24} h$, the area under the curve within $24 h$; AUC, the area under the curve; $T_{\max }$, the time to peak concentration; $T_{1 / 2}$, the eliminate half-life; $C_{\max }$, the peak concentration; $C L_{b}$, relative total systemic clearance; $M R T$, the mean residence time; $F$, the bioavailability.
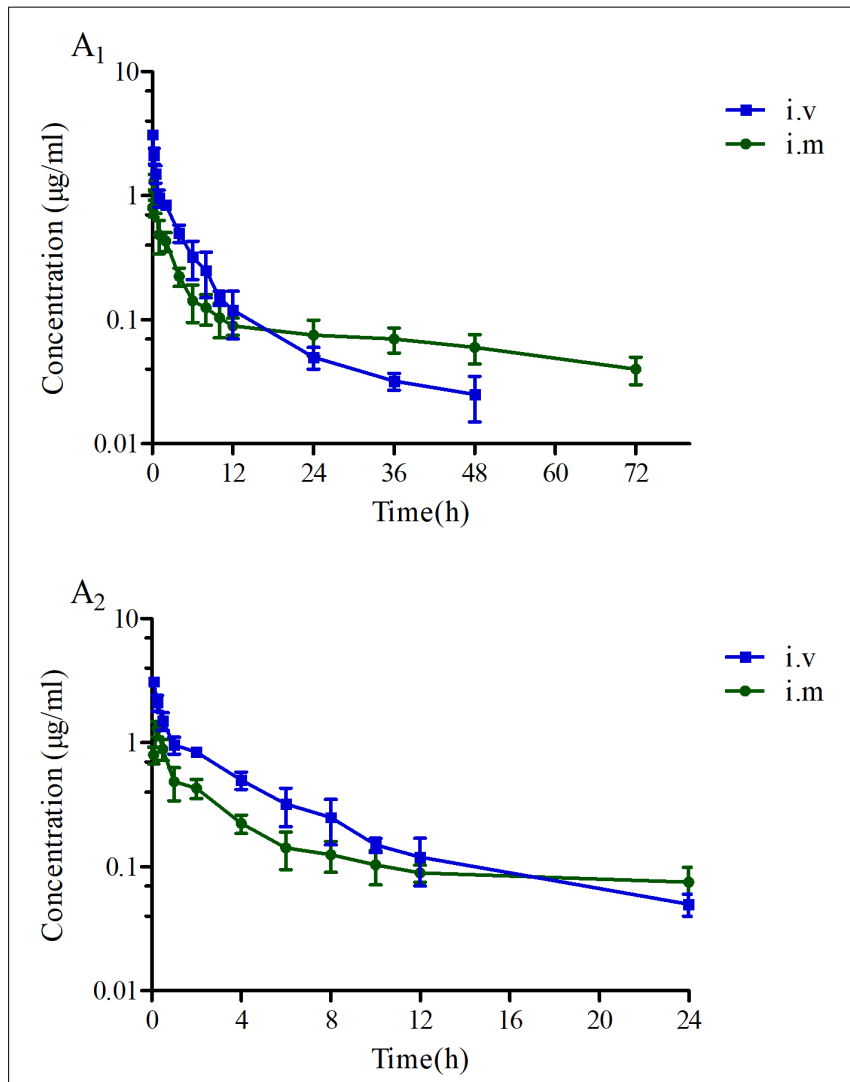

FIGURE 4 | The concentration-time curves of tildipirosin at a dose of $4 \mathrm{mg} / \mathrm{kg}$ after i.v. and i.m. administrations. $\left(\mathbf{A}_{\mathbf{1}}\right)$ Presented the concentration-time during the whole process, $\left(\mathbf{A}_{\mathbf{2}}\right)$ presented the concentration-time within $24 \mathrm{~h}$.

\section{PK/PD Integration and Modeling}

As a concentration-dependent antibiotic, the selected PK/PD parameters attained from PK data in vivo combined with MIC ex vivo were presented in the Table 3 . The ratios of $C_{\max } / \mathrm{MIC}$, $\mathrm{AUC}_{24} \mathrm{~h} / \mathrm{MIC}$ and $\mathrm{T}>\mathrm{MIC}$ were $1.96,7.88$, and $0.97 \mathrm{~h}$, respectively, on the basis of $\mathrm{PK} / \mathrm{PD}$ data ex vivo (Table 2). Ex vivo antibacterial activity of tildipirosin against PM04 was measured in serum samples collected before and at $0.25,0.5,1$,
TABLE 3 | The parameters of PK/PD integration of tildipirosin.

\begin{tabular}{lcc}
\hline Parameters & Units & Mean \pm SD \\
\hline $\mathrm{AUC}_{24}{ }_{h} / \mathrm{MIC}$ & $\mathrm{h}$ & $7.88 \pm 0.87$ \\
$C_{\max } / \mathrm{MIC}$ & - & $1.96 \pm 0.21$ \\
$T>\mathrm{MIC}$ & $\mathrm{h}$ & $0.97 \pm 0.10$
\end{tabular}

TABLE 4 | The main ex vivo parameters of PK/PD modeling of tildipirosin in plasma.

\begin{tabular}{lcc}
\hline Parameters & Units & Mean $\pm \mathbf{S D}$ \\
\hline$E_{\max }$ & LgCFU/ml & $2.54 \pm 0.37$ \\
$\mathrm{EC}_{50}$ & $\mathrm{~h}$ & $23.52 \pm 1.80$ \\
$N$ & - & $3.48 \pm 0.81$ \\
$\mathrm{AUC}_{24 \mathrm{~h}} / \mathrm{MIC}$ for bacteriostatic $(E=0)$ & $\mathrm{h}$ & $18.91 \pm 1.32$ \\
$\mathrm{AUC}_{24 \mathrm{~h}} / \mathrm{MIC}$ for bactericidal $(E=-3)$ & $\mathrm{h}$ & $29.13 \pm 2.44$ \\
$\mathrm{AUC}_{24 \mathrm{~h}} / \mathrm{MIC}$ for eradication $(E=-4)$ & $\mathrm{h}$ & $34.03 \pm 4.11$
\end{tabular}

$E_{\text {max }}$, presented the $\mathrm{Lg}$ change in bacterial counts of blank sample; $E C_{50}$, presented the value to achieve $50 \%$ maximal antibacterial effect; $N$, presented the Hill coefficient.

$2,4,12$, and $24 \mathrm{~h}$ after i.m. induction. The relationship between antimicrobial efficiency and the ex vivo PK/PD parameter of $\mathrm{AUC}_{24}{ }_{h} / \mathrm{MIC}$ ratios were simulated by using the inhibitory sigmoid $E_{\max }$ model. The model parameters of the Hill coefficient $\mathrm{N}, E_{\max }$, and $\mathrm{AUC}_{24} h / \mathrm{MIC}$ values are presented for three levels of growth inhibition in Table 4 and Figure 5. The values of the $\mathrm{AUC}_{24}{ }_{h} / \mathrm{MIC}$ ratio required for bacteriostatic activity $(E=0)$, bactericidal activity $(E=-3)$, and bacterial elimination $(E=-4)$ were 18.91, 29.13, and $34.03 \mathrm{~h}$, as presented in Table 4.

\section{PK/PD Cutoff Calculation of Tildipirosin Against PM04}

The calculated cumulative target achievement for PK/PD parameter $\left(\mathrm{AUC}_{24} \mathrm{~h} / \mathrm{MIC}\right.$ ) in serum (ex vivo) was $29.13 \pm 2.44 \mathrm{~h}$, with assured bactericidal activity $(E=-3)$. The PTA values were calculated as $0,0,68.64$, and $100 \%$ when the MIC values were defined as $0.5,0.25,0.125$, and $0.0625 \mu \mathrm{g} / \mathrm{ml}$, respectively, after a single $4 \mathrm{mg} / \mathrm{kg}$ i.m. was administrated in pigs (Table 5). As a result, a PTA $\geq 90 \%$ could be achieved for isolates with MIC $\leq 0.0625 \mu \mathrm{g} / \mathrm{ml}$ in the serum after i.m. injection at a dose of $4 \mathrm{mg} / \mathrm{kg}$ body weight (Table 5). Furthermore, it could be deduced to be $0.25 \mu \mathrm{g} / \mathrm{ml}$ in TSB (in vitro). Therefore, the $\mathrm{CO}_{\mathrm{PD}}$ of tildipirosin against PM could be defined as $0.25 \mu \mathrm{g} / \mathrm{ml}$ in vitro.

\section{Estimation of Dosages}

The predicted daily doses were given in Table 6 based on $\mathrm{AUC}_{24 \mathrm{~h}} / \mathrm{MIC}$ ratios and $\mathrm{CL}_{\mathrm{b}}$ for these three levels of antibacterial activity measured from the PK/PD integrating model and the distribution of ex vivo MIC using Monte Carlo Simulations in Oracle Crystal Ball. The distributions of predicted population dose $\left(\mathrm{AUC}_{24} \mathrm{~h} / \mathrm{MIC}\right)$ values of tildipirosin curing PM for 50 and $90 \%$ targets were observed, respectively, and illustrated in Figure 6. In this research, based on the dose equations, the predicted doses for bacteriostatic, bactericidal, and elimination activity of tildipirosin against PM over $24 \mathrm{~h}$ were $6.10,9.41$, and 


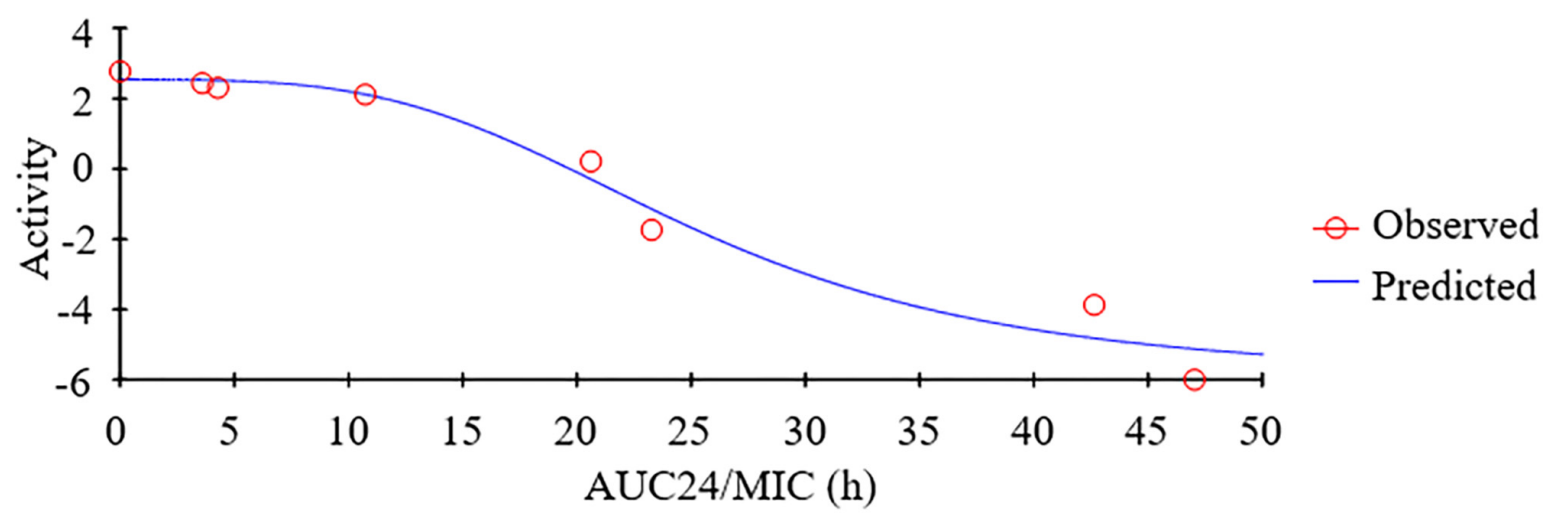

FIGURE 5 | Plots of ex vivo $\mathrm{AUC}_{24} \mathrm{~h} / \mathrm{MIC}$ ratios versus the amount of difference within $24 \mathrm{~h}$.

TABLE 5 | The $\mathrm{AUC}_{24} / \mathrm{MIC}$ values calculated with Monte Carlo simulation for PTA.

\begin{tabular}{llllll}
\hline Doses & Effect & \multicolumn{5}{c}{ MIC $(\boldsymbol{\mu g} / \mathbf{m l})$} \\
\hline $4 \mathrm{mg}$ & Eradication & $\mathbf{0 . 0 6 2 5 ^ { * }}$ & $\mathbf{0 . 1 2 5}$ & $\mathbf{0 . 2 5}$ & $\mathbf{0 . 5}$ \\
\hline
\end{tabular}

* Represents the value of PKJPD cutoff breakpoint.

TABLE 6 | The predicted daily doses of tildipirosin curing PM.

\begin{tabular}{lcc}
\hline Predicted doses $(\mathbf{m g} / \mathbf{k g} \cdot \mathbf{b w})$ & \multicolumn{2}{c}{ Target ratios } \\
\cline { 2 - 3 } & $\mathbf{5 0 \%}$ & $\mathbf{9 0 \%}$ \\
\hline Bacteriostatic $(E=0)$ & 6.10 & 7.86 \\
Bactericidal $(E=-3)$ & 9.41 & 12.17 \\
Eradication $(E=-4)$ & 10.96 & 14.57 \\
\hline
\end{tabular}

$10.96 \mathrm{mg} / \mathrm{kg}$.bw for $50 \%$ target, respectively, and $7.86,12.17$, and $14.57 \mathrm{mg} / \mathrm{kg}$.bw for $90 \%$ target, respectively, in Table 6.

\section{DISCUSSION}

As the newest macrolide antibiotic, tildipirosin has a longacting, strong bacteriostatic action, high bioavailability and drug concentrations particularly in lung tissue and other outstanding profiles (Rose et al., 2013; Lei et al., 2018a). In the current study, the MICs of 112 clinical isolates (PM) from across the country of China were monitored according to the CLSI M07-A9 guidance document. The MIC concentrations of tildipirosin against PM were in the range of $0.0625-32 \mu \mathrm{g} / \mathrm{ml}$ containing three resistant bacteria with MICs over or equal to $16 \mu \mathrm{g} / \mathrm{ml}$, in possession of resistance genes erm (42) or msr (E) and mph (E) in this study. These resistant genes for PM to tildipirosin have been demonstrated in the previously described reports by Jacob, Lei, and Geovana, respectively (Michael et al., 2012; Poehlsgaard et al., 2012; Lei et al., 2018a). The resistance of PM to macrolides including tildipirosin was considered as more and more serious. Furthermore, the control of PM was difficult due to its high resistance and rapid spread to common antimicrobial drugs resulting from the overuse and misuse (Tang et al., 2009; Ferreira et al., 2012). Therefore, it is necessary to establish an optimal scheme to effectively reduce the PM resistance development.

In the current study, the $\mathrm{MIC}_{50}$ and $\mathrm{MIC}_{90}$ of the 112 clinical isolated PM across China were tested to be 0.5 and $2 \mu \mathrm{g} / \mathrm{ml}$ in vitro, respectively. The isolated PM04 with the high toxicity gene (toxA) from Anhui province was selected as the respective PM for the further study, and the MIC of the PM0 4 was $2 \mu \mathrm{g} / \mathrm{ml}$ in TSB, similar to the value of $\mathrm{MIC}_{90}$ (Table 1). Additionally, the MIC of the PM04 in vivo (serum) was detected to be $0.5 \mu \mathrm{g} / \mathrm{ml}$ (Table 1), which is 0.25 times lower than that in vitro. The difference (MICs) between TSB and serum might be mainly due to the serum effect which was also reported in the previous studies by Toutain et al. (2016) and Lei et al. (2017b). In most of the previously published reports, the clinical isolates were randomly selected for PK/PD analysis (Robertson et al., 2005; Haritova et al., 2006; Nedelman et al., 2007; Sidhu et al., 2010). However, in this study, the PM04 was the most representative clinical isolate with high toxicity (which has been demonstrated in Kunming mice, but not presented in this study) and high MIC value similar to the $\mathrm{MIC}_{90}$ of the population (PM). Therefore, it could be more reliable for further study.

Based on the guidelines of CLSI, it is usually encouraged that the susceptibility of wild-type bacteria to antibiotics should be tested prior to their use for treatment (Lei et al., 2018a). There has not been any susceptibility breakpoint standard for tildipirosin against PM. The ECV of tildipirosin against PM was calculated to be $4 \mu \mathrm{g} / \mathrm{ml}$ in this study, which was the first declared result and the ECV was calculated using the integrated software "ECOFFinder" which could simplify the procedures and progresses. The ECOFFinder was also applied in the report published by Lees et al. (2015). Compared with the previously published study by Lei et al. (2018a), the ECV of tildipirosin against HPS was $8 \mu \mathrm{g} / \mathrm{ml}$ which is two times higher than that $(4 \mu \mathrm{g} / \mathrm{ml}, \mathrm{PM})$ in this study. The difference of these two bacteria might attribute to the wild-type MIC ranges: the MIC of tildipirosin against HPS was in the range of $0.03125-256 \mu \mathrm{g} / \mathrm{ml}$, while the MIC range of tildipirosin against PM was from 0.06125 to $32 \mu \mathrm{g} / \mathrm{ml}$. Moreover, the species difference might also be another reason. The $\mathrm{CO}_{\mathrm{PD}}$ 
$A_{1}$

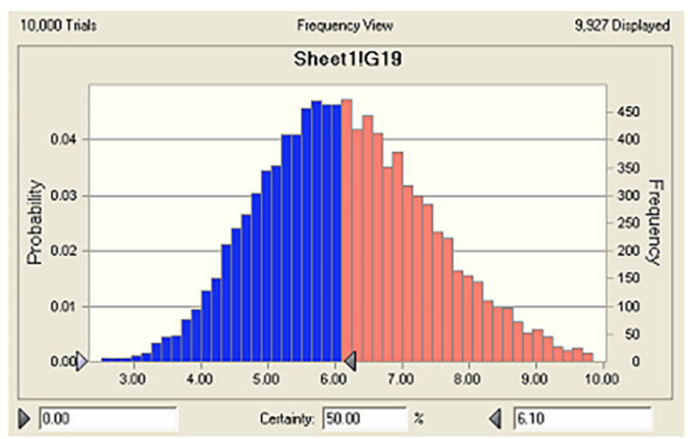

$B_{1}$

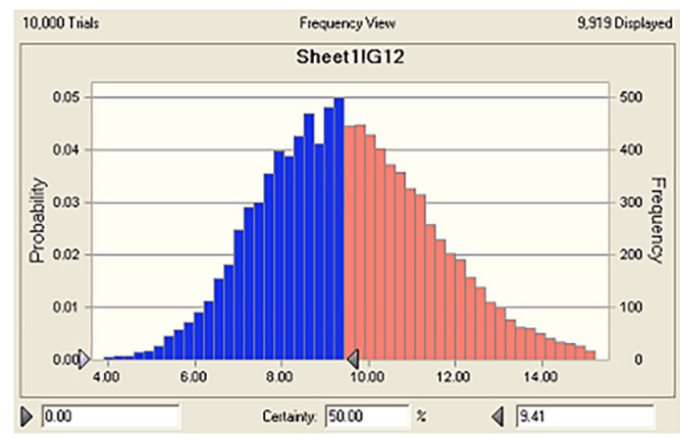

$C_{1}$

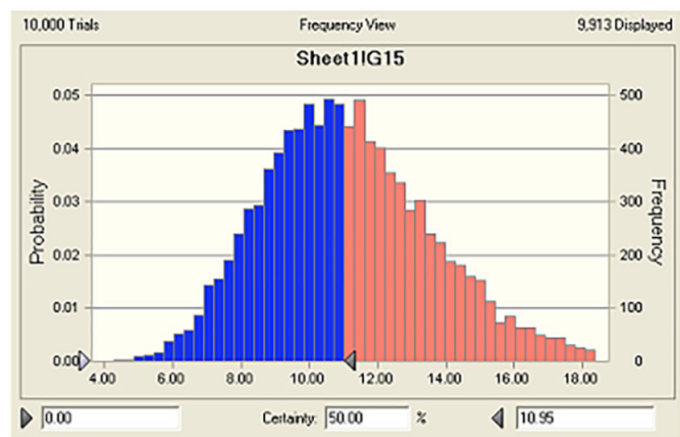

$\mathrm{A}_{2}$

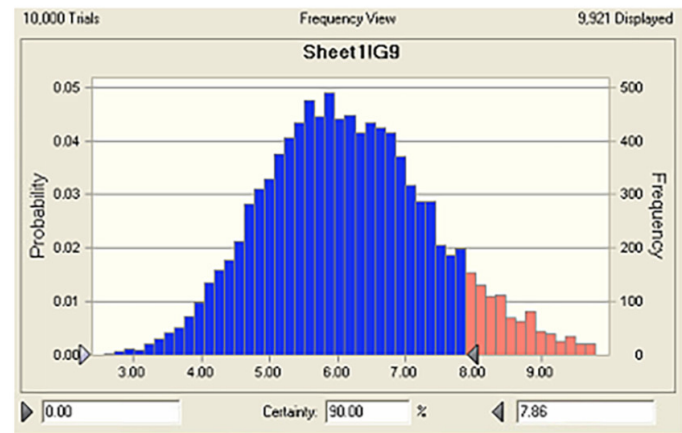

$\mathrm{B}_{2}$

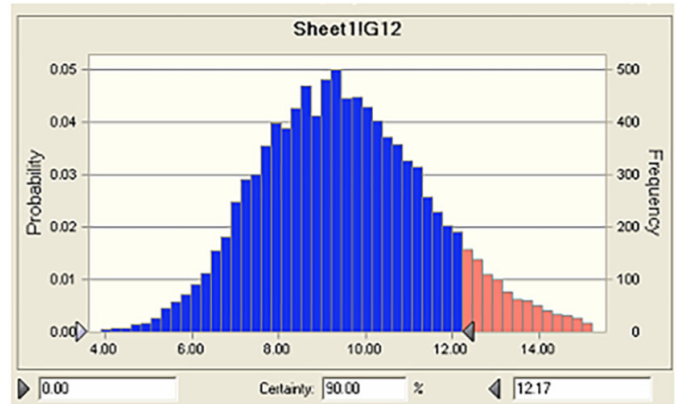

$\mathrm{C}_{2}$

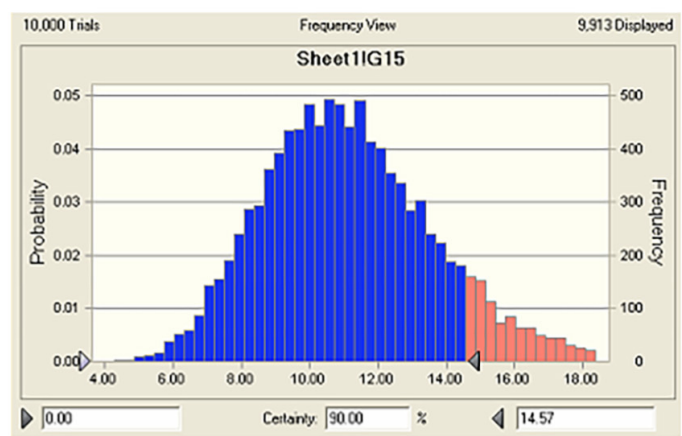

FIGURE 6 | The predicted regimes of tildipirosin curing PM for 50\% and 90\% TAR. $\left(\mathbf{A}_{\mathbf{1}}-\mathbf{C}_{\mathbf{1}}\right)$ Meant the predicted population doses for bacteriostatic, bactericidal, and elimination activities for 50\% TAR; $\left(\mathbf{A}_{2}-\mathbf{C}_{2}\right)$ meant the predicted population doses for bacteriostatic, bactericidal, and elimination activities for $90 \%$ TAR.

of tildipirosin against PM was calculated to be $0.25 \mu \mathrm{g} / \mathrm{ml}$ in the TSB (Table 5). Although the susceptibility breakpoint was defined based on the three cutoff values including ECV, $\mathrm{CO}_{\mathrm{PD}}$ and clinical cutoff, the last cutoff was required to monitor in the clinic (Toutain et al., 2017). According to the guidelines of CLSI and EUCAST, the final breakpoint could be recognized as $4 \mu \mathrm{g} / \mathrm{ml}$ without the data from clinical investigative findings. The result of susceptibility breakpoint $(4 \mu \mathrm{g} / \mathrm{ml})$ provided from this study could be regarded as an alternative for isolated strains MIC determination in clinical veterinary medicine.
The mean concentrations of tildipirosin over the MIC $(0.5 \mu \mathrm{g} / \mathrm{ml})$ for $\mathrm{PM}$ in the serum $(T>\mathrm{MIC})$ sustained $0.97 \mathrm{~h}$ (Table 3), demonstrating that tildipirosin had a strong and long-lasting bacteriostatic activity against PM which was similar to HPS ( $4 \mathrm{~h}, T>\mathrm{MIC}$ ) in the previous reports (Rose et al., 2013; Lei et al., 2018a). Additionally, the means of the $\mathrm{AUC}_{24} \mathrm{~h}\left(3.94 \mu \mathrm{g}^{*} \mathrm{~h} / \mathrm{ml}\right), C_{\max }(0.98 \mu \mathrm{g} / \mathrm{ml})$, and $\mathrm{CL}_{\mathrm{b}}$ $(0.43 \mathrm{~L} / \mathrm{h})$ in this study were similar to those $\left(4.25 \mu \mathrm{g}^{*} \mathrm{~h} / \mathrm{ml}\right.$, $1.01 \mu \mathrm{g} / \mathrm{ml}$, and $0.28 \mathrm{~L} / \mathrm{h}$ ) in the published report by Lei et al. (2018a). The PK profiles for tildipirosin in pigs after i.v. administration were investigated for the first time, and 
the absolute bioavailability of i.m. administrated tildipirosin was calculated to be $85.5 \%$ which was similar to the study on the cattle (78.9\%) reported by Menge et al. (2012) and Rose et al. (2013). The serum protein binding ratio was tested to be $22 \%$ which was similar to the report of EMA (30\%) (EMA, 2010). These results demonstrated that tildipirosin had a high extent of absorption and low-level of protein binding effect and could be proposed widely for use in the veterinary clinic practice.

Based on the killing-time curve profiles of tildipirosin against PM04 (Figure 3) in vitro and ex vivo, it was obvious that tildipirosin presented the concentration-dependent action and the parameter " $\mathrm{AUC}_{24 \mathrm{~h}} / \mathrm{MIC}$ " was generally regarded as the threshold for the successful therapeutic outcome of macrolides (Lei et al., 2017b, 2018b,c). In the previously published studies (Sang et al., 2016; Wang et al., 2016; Zhang et al., 2016; Lei et al., 2017a, 2018a), the $\mathrm{AUC}_{24} \mathrm{~h} / \mathrm{MIC}>30 \mathrm{~h}$, and $\mathrm{AUC}_{24 \mathrm{~h}} / \mathrm{MIC}>125 \mathrm{~h}$ were used frequently for macrolides and fluoroquinolones against Gram-negative bacteria; these thresholds might be different for different drugs, against different kinds of bacteria due to the differences in the immune status of target animals and pathogens (Toutain et al., 2002; Ahmad et al., 2015). Therefore, it is essential to obtain the exclusive PK/PD target for tildipirosin against PM (Lei et al., 2018c). In this study, the PK/PD target ( $\mathrm{AUC}_{24 \mathrm{~h}} / \mathrm{MIC}$ ) for tildipirosin against PM was $29.13 \mathrm{~h}$ when it acted on the bactericidal activity $(E=-3)$ using $\mathrm{PK} / \mathrm{PD}$ integration modeling (Table 4). This target value was much higher than the original parameter $(7.88 \mathrm{~h})\left(\mathrm{AUC}_{24 \mathrm{~h}} / \mathrm{MIC}\right)$ in Table 3; this result revealed that the current dosage ( $4 \mathrm{mg} / \mathrm{kg}$ ) might not reach the bactericidal effect of tildipirosin against PM. Therefore, the optimal dosage regimens are extremely necessary. Based on the ex vivo PK/PD modeling using inhibitory sigmoidal $E_{\max }$ model, a favorable correlation $\left(R^{2}=0.988\right)$ was shown between the PK/PD index $\left(\mathrm{AUC}_{24 \mathrm{~h}} / \mathrm{MIC}\right)$ and predicted antibacterial efficacy which could be observed in Figure 5. Furthermore, the predicted population daily dosages over $24 \mathrm{~h}$ for tildipirosin against PM acting bacteriostatic, bactericidal, and eradication activities were calculated as 6.10, 9.41, and $10.96 \mathrm{mg} / \mathrm{kg}$ for $50 \% \mathrm{TAR}$, and $7.86,12.17$ and $14.57 \mathrm{mg} / \mathrm{kg}$ TAR, respectively (Figure 6 and Table 6) according to the Monte Carlo simulation which is an effective method to adjust the dosage regimen for clinical use (Nielsen and Friberg, 2013; Dorey et al., 2017). Therefore, it was suggested that $12.17 \mathrm{mg} / \mathrm{kg}$ could guarantee the clinical efficacy and bactericidal action in this study. Due to the bacterial endpoint, in vivo might differ from the predicted dosages and the target animals' immune system

\section{REFERENCES}

Ahmad, I., Hao, H., Huang, L., Sanders, P., Wang, X., Chen, D., et al. (2015). Integration of PK/PD for dose optimization of Cefquinome against Staphylococcus aureus causing septicemia in cattle. Front. Microbiol. 6:588. doi: 10.3389/fmicb. 2015.00588

Aliabadi, F. S., and Lees, P. (2000). Antibiotic treatment for animals: effect on bacterial population and dosage regimen optimisation. Int. J. Antimicrob. Agents 14, 307-313. doi: 10.1016/S0924-8579(00) 00142-4

Aliabadi, F. S., and Lees, P. (2001). Pharmacokinetics and pharmacodynamics of danofloxacin in serum and tissue fluids of goats following intravenous and could also affect the bacterial eradication action of drug, and the collected animals' samples (a small scale) in this study were not enough to strongly support the conclusions. The estimated population daily dosages should be validated in future for veterinary clinical practice and research.

\section{CONCLUSION}

The intelligent use of antibiotics was increasingly important in the veterinary clinic, and the misuse and abuse of antibiotics were the uppermost reason for the bacteria resistance development (Nguyen et al., 2012; Lei et al., 2018c). The current study firstly provided the susceptibility breakpoint $(4 \mu \mathrm{g} / \mathrm{ml})$ which could distinguish the resistance bacteria easily and clearly and be regarded as a kind of resistance standard for tildipirosin against PM in further studies. Furthermore, the finding of this study also proved that the current dosage $4 \mathrm{mg} / \mathrm{kg}$ tildipirosin $\left(\mathrm{AUC}_{24} \mathrm{~h} / \mathrm{MIC}=7.88 \mathrm{~h}\right)$ could not cover and guarantee the bactericidal effect, while the predicted daily dosage over $24 \mathrm{~h}$ $(12.17 \mathrm{mg} / \mathrm{kg}$ ) might be sufficient and effective (Table 6). In conclusion, the susceptibility breakpoint and predicted daily dosage from this study have to be further validated in clinical practice.

\section{AUTHOR CONTRIBUTIONS}

JC and $\mathrm{QH}$ conceived this study. QL and ZL designed the experiments. ZL, YQ, KL, HZ, and BY performed the experiments. ZL wrote the manuscript. QH, HK, SA, JX, GM, $\mathrm{WZ}$, and JC improved the language. All authors reviewed the manuscript.

\section{FUNDING}

This work was partly supported by China's Agricultural Research System (CARS-36).

\section{ACKNOWLEDGMENTS}

We thank Huazhong Agricultural University for permission to conduct this research.

intramuscular administration. Am. J. Vet. Res. 62, 1979-1989. doi: 10.2460/ajvr. 2001.62.1979

Aliabadi, F. S., and Lees, P. (2002). Pharmacokinetics and pharmacodynamic integration of marbofloxacin in calf serum, exudates and transudate. J. Vet. Pharmacol. Ther. 25, 161-174. doi: 10.1046/j.1365-2885.2002.00399.x

Andersen, N. M., Poehlsgaard, J., Warrass, R., and Douthwaite, S. (2012). Inhibition of protein synthesis on the ribosome by tildipirosin compared with other veterinary macrolides. Antimicrob. Agents Chemother. 56, 6033-6036. doi: 10.1128/AAC.01250-12

Burgess, D. S. (1999). Pharmacodynamic principles of antimicrobial therapy in the prevention of resistance. Chest 115, 19S-23S. doi: 10.1378/chest.115. suppl_1.19S 
Burgess, D. S., Summers, K. K., and Hardin, T. C. (1999). Pharmacokinetics and pharmacodynamics of aztreonam administered by continuous intravenous infusion. Clin. Ther. 21, 1882-1889. doi: 10.1016/S0149-2918(00)86736-3

Chigutsa, E., Meredith, S., Wiesner, L., Padayatchi, N., Harding, J., Moodley, P., et al. (2012). Population pharmacokinetics and pharmacodynamics of ofloxacin in South African patients with multidrug-resistant tuberculosis. Antimicrob. Agents Chemother. 56, 3857-3863. doi: 10.1128/AAC.000 48-12

CLSI (2012). The Methods for Dilution Antimicrobial Susceptibility Determination. Wayne, PA: CLSI.

Dorey, L., Pelligand, L., Cheng, Z., and Lees, P. (2017). Pharmacokinetic/pharmacodynamic integration and modelling of oxytetracycline for the porcine pneumonia pathogens Actinobacillus pleuropneumoniae and Pasteurella multocida. J. Vet. Pharmacol. Ther. 40, 505-516. doi: 10.1111/jvp.12385

Elazab, S. T., Schrunk, D. E., Griffith, R. W., Ensley, S. M., Dell'anna, G., Mullin, K., et al. (2018). Pharmacokinetics of cefquinome in healthy and Pasteurella multocida-infected rabbits. J. Vet. Pharmacol. Ther. 41, 374-377. doi: 10.1111/ jvp. 12489

EMA (2010). Committee for Medicinal Products for Veterinary Use. Available at: http://www.ema.europa.eu/docs/en_GB/document_library/Maximum_Residue _Limits_-_Report/2010/10/WC500097539.pdf

Ferreira, T. S., Felizardo, M. R., Dd, S. D. G., Gomes, C. R., Nogueira Filsner, P. H., Moreno, M., et al. (2012). Virulence genes and antimicrobial resistance profiles of Pasteurella multocida strains isolated from rabbits in Brazil. Sci. World J. 2012:685028. doi: 10.1100/2012/685028

Haritova, A. M., Rusenova, N. V., Parvanov, P. R., Lashev, L. D., and Finkgremmels, J. (2006). Integration of pharmacokinetic and pharmacodynamic indices of marbofloxacin in turkeys. Antimicrob. Agents Chemother. 50, 3779-3785. doi: 10.1128/AAC.00711-05

Holst, E., Rollof, J., Larsson, L., and Nielsen, J. P. (1992). Characterization and distribution of Pasteurella species recovered from infected humans. J. Clin. Microbiol. 30, 2984-2987.

Jian, S., Xia, X., Huang, R. J., Tao, Y., Yi, C., Xi, F., et al. (2015). In vitro dynamic pharmacokinetic/pharmacodynamic (PK/PD) study and COPD of marbofloxacin against Haemophilus parasuis. BMC Vet. Res. 11:293. doi: 10. 1186/s12917-015-0604-5

Lees, P., Pelligand, L., Illambas, J., Potter, T., Lacroix, M., Rycroft, A., et al. (2015). Pharmacokinetic/pharmacodynamic integration and modelling of amoxicillin for the calf pathogens Mannheimia haemolytica and Pasteurella multocida. J. Vet. Pharmacol. Ther. 41, 457-470. doi: 10.1111/jvp.12207

Lei, Y., Xie, S., Chen, D., Pan, Y., Tao, Y., Wei, Q., et al. (2017a). Pharmacokinetic and pharmacodynamic modeling of cyadox against Clostridium perfringens in swine. Sci. Rep. 7:4064. doi: 10.1038/s41598-017-03970-9

Lei, Z., Liu, Q., Xiong, J., Yang, B., Yang, S., Zhu, Q., et al. (2017b). Pharmacokinetic and pharmacodynamic evaluation of marbofloxacin and PK/PD modeling against Escherichia coli in pigs. Front. Pharmacol. 8:542. doi: 10.3389/fphar. 2017.00542

Lei, Z., Liu, Q., Yang, B., Ahmed, S., Cao, J., and He, Q. (2018a). The pharmacokinetic-pharmacodynamic modeling and cut-off values of tildipirosin against Haemophilus parasuis. Oncotarget 9, 1673-1690. doi: 10.18632/ oncotarget. 23018

Lei, Z., Liu, Q., Yang, B., Ahmed, S., Xiong, J., Song, T., et al. (2017c). Evaluation of bioequivalence of two long-acting $20 \%$ oxytetracycline formulations in pigs. Front. Vet. Sci. 4:61. doi: 10.3389/fvets.2017.00061

Lei, Z., Liu, Q., Yang, B., Khaliq, H., Ahmed, S., Fan, B., et al. (2018b). Evaluation of marbofloxacin in beagle dogs after oral dosing: preclinical safety evaluation and comparative pharmacokinetics of two different tablets. Front. Pharmacol. 9:306. doi: 10.3389/fphar.2018.00306

Lei, Z., Liu, Q., Yang, S., Yang, B., Khaliq, H., Li, K., et al. (2018c). PK-PD integration modeling and cutoff value of florfenicol against Streptococcus suis in pigs. Front. Pharmacol. 9:2. doi: 10.3389/fphar.2018.00002

Menge, M., Rose, M., Bohland, C., Zschiesche, E., Kilp, S., Metz, W., et al. (2012). Pharmacokinetics of tildipirosin in bovine plasma, lung tissue, and bronchial fluid (from live, nonanesthetized cattle). J. Vet. Pharmacol. Ther. 35, 550-559. doi: 10.1111/j.1365-2885.2011.01349.x

Michael, G. B., Eidam, C., Kadlec, K., Meyer, K., Sweeney, M. T., Murray, R. W., et al. (2012). Increased MICs of gamithromycin and tildipirosin in the presence of the genes erm(42) and $\operatorname{msr}(\mathrm{E})-\mathrm{mph}(\mathrm{E})$ for bovine Pasteurella multocida and Mannheimia haemolytica. J. Antimicrob. Chemother. 67, 1555-1557. doi: 10.1093/jac/dks076

Mouton, J. W., Dudley, M. N., Cars, O., Derendorf, H., and Drusano, G. L. (2002). Standardization of pharmacokinetic/pharmacodynamic (PK/PD) terminology for anti-infective drugs: an update. Int. J. Antimicrob. Agents 19, 355-358. doi: 10.1016/S0924-8579(02)00031-6

Nedelman, J. R., Rubin, D. B., and Sheiner, L. B. (2007). Diagnostics for confounding in PK/PD models for oxcarbazepine. Stat. Med. 26, 290-308. doi: $10.1002 / \operatorname{sim} .2542$

Nguyen, T. T., Chachaty, E., Huy, C., Cambier, C., De, G. J., Mentré, F., et al. (2012). Correlation between fecal concentrations of ciprofloxacin and fecal counts of resistant Enterobacteriaceae in piglets treated with ciprofloxacin: toward new means to control the spread of resistance? Antimicrob. Agents Chemother. 56, 4973-4975. doi: 10.1128/AAC.06402-11

Nielsen, E. I., and Friberg, L. E. (2013). Pharmacokinetic-pharmacodynamic modeling of antibacterial drugs. Pharmacol. Rev. 65, 1053-1090. doi: 10.1124/ pr.111.005769

Olsen, A. S., Warrass, R., and Douthwaite, S. (2015). Macrolide resistance conferred by rRNA mutations in field isolates of Mannheimia haemolytica and Pasteurella multocida. J. Antimicrob. Chemother. 70, 420-423. doi: 10.1093/jac/dku385

Pfaller, M. A., Andes, D., Diekema, D. J., Espinelingroff, A., and Sheehan, D. (2010). Wild-type MIC distributions, epidemiological cutoff values and species-specific clinical breakpoints for fluconazole and Candida: time for harmonization of CLSI and EUCAST broth microdilution methods. Drug Resist. Updat. 13, 180-195. doi: 10.1016/j.drup.2010.09.002

Pfaller, M. A., Castanheira, M., Diekema, D. J., Messer, S. A., and Jones, R. N. (2011). Triazole and echinocandin MIC distributions with epidemiological cutoff values for differentiation of wild-type strains from non-wild-type strains of six uncommon species of Candida. J. Clin. Microbiol. 49, 3800-3804. doi: 10.1128/JCM.05047-11

Poehlsgaard, J., Andersen, N. M., Warrass, R., and Douthwaite, S. (2012). Visualizing the 16-membered ring macrolides tildipirosin and tilmicosin bound to their ribosomal site. ACS Chem. Biol. 7, 1351-1355. doi: 10.1021/cb300105p

Robertson, S. A., Lascelles, B. D. X., Taylor, P. M., and Sear, J. W. (2005). PKPD modeling of buprenorphine in cats: intravenous and oral transmucosal administration. J. Vet. Pharmacol. Ther. 28, 453-460. doi: 10.1111/j.1365-2885. 2005.00677.x

Rose, M., Menge, M., Bohland, C., Zschiesche, E., Wilhelm, C., Kilp, S., et al. (2013). Pharmacokinetics of tildipirosin in porcine plasma, lung tissue, and bronchial fluid and effects of test conditions on in vitro activity against reference strains and field isolates of Actinobacillus pleuropneumoniae. J. Vet. Pharmacol. Ther. 36, 140-153. doi: 10.1111/j.1365-2885.2012.01397.x

Sang, K., Hao, H., Huang, L., Wang, X., and Yuan, Z. (2016). Pharmacokineticpharmacodynamic modeling of enrofloxacin against Escherichia coli in broilers. Front. Vet. Sci. 2:80. doi: 10.3389/fvets.2015.00080

Sidhu, P. K., Landoni, M. F., Aliabadi, F. S., and Lees, P. (2010). PK-PD integration and modeling of marbofloxacin in sheep. Res. Vet. Sci. 88, 134-141. doi: 10. 1016/j.rvsc.2009.05.013

Sidhu, P. K., Landoni, M. F., Aliabadi, M. H. S., Toutain, P. L., and Lees, P. (2011). Pharmacokinetic and pharmacodynamic modelling of marbofloxacin administered alone and in combination with tolfenamic acid in calves. J. Vet. Pharmacol. Ther. 34, 376-387. doi: 10.1111/j.1365-2885.2010.01247.x

Tang, X., Zhao, Z., Hu, J., Wu, B., Cai, X., He, Q., et al. (2009). Isolation, antimicrobial resistance, and virulence genes of Pasteurella multocida strains from swine in China. J. Clin. Microbiol. 47, 951-958. doi: 10.1128/JCM. 02029-08

Torres, F., Santamaria, R., Jimenez, M., Menjón, R., Ibanez, A., Collell, M., et al. (2016). Pharmacokinetics of tildipirosin in pig tonsils. J. Vet. Pharmacol. Ther. 39, 199-201. doi: 10.1111/jvp.12258

Toutain, P. L., Bousquetmélou, A., Damborg, P. P., Ferran, A. A., Mevius, D., Pelligand, L., et al. (2017). En route towards European clinical breakpoints for veterinary antimicrobial susceptibility testing: a position paper explaining the VetCAST approach. Front. Microbiol. 8:2344. doi: 10.3389/fmicb.2017. 02344

Toutain, P. L., Castillo, J. R. E. D., and Bousquet-Mélou, A. (2002). The pharmacokinetic-pharmacodynamic approach to a rational dosage regimen for antibiotics. Res. Vet. Sci. 73, 105-114. doi: 10.1016/S0034-5288(02)00039-5 
Toutain, P. L., Potter, T., Pelligand, L., Lacroix, M., Illambas, J., and Lees, P. (2016). Standard PK/PD concepts can be applied to determine a dosage regimen for a macrolide: the case of tulathromycin in the calf. J. Vet. Pharmacol. Ther. 40, 16-27. doi: 10.1111/jvp.12333

Turnidge, J., and Paterson, D. L. (2007). Setting and revising antibacterial susceptibility breakpoints. Clin. Microbiol. Rev. 20, 391-408. doi: 10.1128/CMR. 00047-06

Wang, J., Hao, H., Huang, L., Liu, Z., Chen, D., and Yuan, Z. (2016). Pharmacokinetic and pharmacodynamic integration and modeling of enrofloxacin in swine for Escherichia coli. Front. Microbiol. 7:36. doi: $10.3389 /$ fmicb. 2016.00036

Xiao, X., Sun, J., Chen, Y., Huang, R. J., Huang, T., Qiao, G. G., et al. (2015). In vitro dynamic pharmacokinetic/pharmacodynamic(PK/PD) modeling and PK/PD cutoff of cefquinome against Haemophilus parasuis. BMC Vet. Res. 11:33. doi: 10.1186/s12917-015-0343-7

Zhang, P., Hao, H., Li, J., Ahmad, I., Cheng, G., Chen, D., et al. (2016). The epidemiologic and pharmacodynamic cutoff values of tilmicosin against
Haemophilus parasuis. Front. Microbiol. 7:385. doi: 10.3389/fmicb.2016.0 0385

Zhixin, L., Shulin, F., Bing, Y., Qianying, L., Saeed, A., Lei, X., et al. (2017). Comparative transcriptional profiling of tildipirosin-resistant and sensitive Haemophilus parasuis. Sci. Rep. 7:7517. doi: 10.1038/s41598-017-07972-5

Conflict of Interest Statement: The authors declare that the research was conducted in the absence of any commercial or financial relationships that could be construed as a potential conflict of interest.

Copyright (C) 2018 Lei, Liu, Qi, Yang, Khaliq, Xiong, Moku, Ahmed, Li, Zhang, Zhang, $\mathrm{Cao}$ and $\mathrm{He}$. This is an open-access article distributed under the terms of the Creative Commons Attribution License (CC BY). The use, distribution or reproduction in other forums is permitted, provided the original author(s) and the copyright owner(s) are credited and that the original publication in this journal is cited, in accordance with accepted academic practice. No use, distribution or reproduction is permitted which does not comply with these terms. 